\title{
Structured extended finite element methods for solids defined by implicit surfaces
}

\author{
Ted Belytschko ${ }^{1, * \dagger}$, Chandu Parimi ${ }^{1}$, Nicolas Moës $^{2}$, N. Sukumar ${ }^{3}$ and Shuji Usui ${ }^{1}$ \\ ${ }^{1}$ Department of Mechanical Engineering, Northwestern University, Evanston, IL 60208, U.S.A. \\ ${ }^{2}$ Ecole Centrale de Nantes, Nantes F-44321, France \\ ${ }^{3}$ Department of Civil and Environmental Engineering, UC Davis, Davis CA 95616, U.S.A.
}

\begin{abstract}
SUMMARY
A paradigm is developed for generating structured finite element models from solid models by means of implicit surface definitions. The implicit surfaces are defined by radial basis functions. Internal features, such as material interfaces, sliding interfaces and cracks are treated by enrichment techniques developed in the extended finite element method. Methods for integrating the weak form for such models are proposed. These methods simplify the generation of finite element models. Results presented for several examples show that the accuracy of this method is comparable to standard unstructured finite element methods. Copyright (C) 2002 John Wiley \& Sons, Ltd.
\end{abstract}

KEY WORDS: finite elements; structured meshes; unit cells; representative volume elements; interfaces

\section{INTRODUCTION}

Meshing of three-dimensional solids is still one of the most burdensome tasks in finite element analysis. The difficulties of meshing have become particularly acute with the emergence of models with $10^{7}$ to $10^{9}$ elements. In treating such large-scale, unstructured finite element meshes, an inordinate amount of effort is devoted to:

- generating the mesh

- coping with the unstructured character of the equations during assembly and solution procedures

- post-processing

Recently, it has become apparent that many of these difficulties can be circumvented by using structured meshes in conjunction with recently developed techniques for representing

\footnotetext{
* Correspondence to: Ted Belytschko, Department of Mechanical Engineering, Northwestern University, 2145 Sheridan Road, Evanston, IL 60208, U.S.A.

†E-mail: tedbelytschko@northwestern.edu
}

Contract/grant sponsor: Office of Naval Research and Army Research Office

Copyright (c) 2002 John Wiley \& Sons, Ltd. 
internal discontinuities [1], and internal details [2]. In fact, with these techniques, it becomes possible to model the detail associated with engineering problems with even greater fidelity than conventional finite element methods. For example, it is possible to model complex sliding surfaces within a body and to model cracks and small holes.

One of the sources from which these capabilities have evolved is the seminal paper by Melenk and Babuska [3], in which the concept of partition of unity was first described. Belytschko and Black [4] employed the concept to model cracks; in Moës et al. [5] and Dolbow et al. [6] step functions were introduced through the partition of unity to model arbitrary discontinuities. They called the method the extended finite element method (X-FEM). Babuska et al. [3] and Strouboulis et al. [7] illustrated the potential of the partition of unity concept in modeling small holes in a mesh and introducing so-called handbook solutions; they called it the generalized finite element method. The method was expanded in Strouboulis et al. [2], where the focus was towards the extension of the classical finite element method to meshes that do not conform to boundaries of the problem. In Moës et al. [5] and Belytschko et al. [1] the quadrature issue was studied for meshes that do not conform to internal boundaries.

The proposed method also has some capabilities that would be very difficult to incorporate in an unstructured stress analysis program for solids:

- The ability to model crack growth, such as due to fatigue, without any remeshing.

- The ability to easily model complex systems of sliding interfaces, such as joints in rocks.

A novel feature of the method described here is its use of implicit surface definitions for both external and internal surfaces. This enables the use of powerful techniques for surface definition by implicit functions that have recently been developed; see Carr et al. [8] and O'Brien et al. [9]. In the former, up to $3 \times 10^{6}$ points were used to define extremely complex external surfaces; these large systems were solved by multipolar methods.

In this paper we describe a methodology for constructing the finite elements for structured meshes for objects described by implicit surfaces, both for the outside boundary and interior surfaces. For existing parts, a laser scan of body can be immediately translated into an implicit description of the external boundaries. By means of holography and other methods, implicit function descriptions of any internal surfaces can also be obtained. They can then be translated to finite element models as described here. For CAD models or solid models, the construction of an implicit surface model is also straightforward, for it is only necessary to extract a set of surface points from the geometric model. Thus, the paradigm described here should enable finite element analyses of complex engineering problems with almost no human intervention.

The concept of describing internal surfaces of a problem independent of a mesh by implicit functions originated in Sukumar et al. [10]. It has been used to model crack growth with level sets in two dimensions by Stolarska et al. [11], crack growth in three dimensions by Gravouil et al. [12]. The methodology has also been applied to solidification, Chessa et al. [13] and fluid interfaces, Chessa et al. [14], and for particles in fluids by Wagner et al. [15].

Thus the basis is available for the rapid development of methods that combine implicit surface descriptions of engineering components with structured finite element analysis. As indicated in this paper, the synthesis of these methods makes possible an order of magnitude simplification in the development of finite element discretization for complex solid models.

The outline of this paper is as follows. In the first section, the components of the paradigm, including the implicit surface definitions, the approximating functions and the background 
meshes are introduced. The finite element discretization, the strong form and weak form of the governing equations are then explained, with emphasis on quadrature for integration in the framework of the function definitions. We then look into the internal details such as cracks and material interfaces defined as implicit functions. Results for function definitions and complete solutions are then presented.

\section{CAD MODEL}

\subsection{Overview}

We consider a body $\Omega$ with boundary $\Gamma$. The Euclidean co-ordinates are $\mathbf{x}=[x, y, z]$, where boldface denotes a matrix or vector. We also use indicial notation, with lower case indices pertaining to Cartesian components and upper case indices pertaining to nodes or points. Repeated lower case indices are summed on the number of spacial dimensions $n_{\mathrm{sd}}$.

We will first describe the procedure for the case when the object is enclosed by a single surface. It may be convex or concave. The procedure described here consists of the following steps.

- a set of points $\mathbf{x}^{I}, I=1$ to $n_{\mathrm{sp}}$ on the surface of the object and a few interior points $\mathbf{x}^{J}$, $J=1$ to $n_{\text {ip }}$ (the superscript $I$ denotes index of the points that define the shape of the object) are obtained.

- an implicit function is constructed from $\mathbf{x}^{I}$ such that $\phi\left(\mathbf{x}^{I}\right)=0$ defines the surface of the body.

- a set of voxels for 3D (pixels for 2D) that encompass the entire domain for which $\phi(\mathbf{x}) \leqslant 0$ are constructed.

- a finite element discretization (called active pixels or voxels) is obtained based on the voxels that are encompassed by the implicit surface.

These steps are illustrated in Figure 1. For simplicity, we henceforth refer to both pixels and voxels as voxels with the implicit assumption that in 2D we are refering to pixels.

\subsection{Shape definition by implicit functions}

As can be seen from Figure 1, we start with the set of points that define the object. The points can be taken either directly from the object by a scanner for the analysis of an existing component, or they may be based on a CAD model. We defer the issue of interior features, such as material interfaces, cracks, etc., to later.

The first step is to construct the implicit function description of the body. The surfaces are described by fitting an approximant to a set of points on the surface $\mathbf{x}^{I}, I=1$ to $n_{s p}$. A set of off-surface points $\mathbf{x}^{I}, I=1$ to $n_{i p}$ is also needed; these are generated by moving away from the surface by an approximate surface normal, as in Turk and O'Brien [16].

At this time, we have chosen radial basis functions as the approximants, for the description of surfaces by these techniques has achieved a relatively high state of robustness, see Carr et al. [8] and O'Brien et al. [9] for more detail. Implicit approximations of functions define the surface by

$$
\phi(\mathbf{x})=0
$$



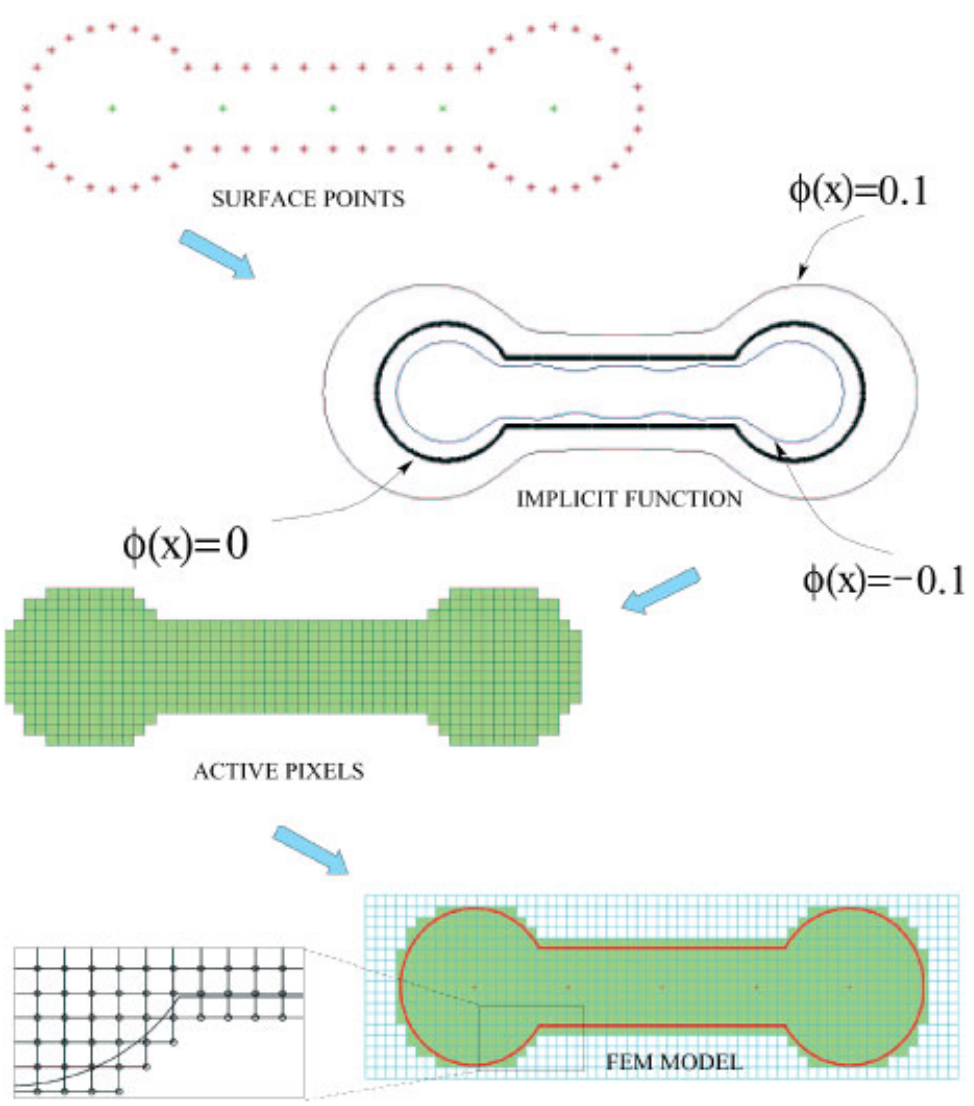

Figure 1. The schema for the CAD/FEM paradigm.

i.e. the surface is the zero isobar of the function $\phi(\mathbf{x})$. We impose the additional conditions:

$$
\begin{array}{ll}
\phi(\mathbf{x})<0 & \text { for } \mathbf{x} \in \Omega \\
\phi(\mathbf{x})>0 & \text { for } \mathbf{x} \notin \Omega
\end{array}
$$

The expression for $\phi(\mathbf{x})$ in terms of the radial basis functions $R(r)$ are

$$
\phi(\mathbf{x})=\alpha_{0}+\alpha_{-j} \mathbf{x}_{j}+\sum_{I=1}^{N} \alpha_{I} R\left\|\mathbf{x}-\mathbf{x}^{I}\right\|
$$

where $\alpha_{I}, I=-n_{S D}$ to $N=n_{i p}+n_{s p}$ are arbitrary parameters chosen to best fit the surface; $r=\left\|x-x^{I}\right\|$. The low order polynomial preceding the sum is added to achieve completeness of the approximation. Examples of radial basis functions are

$$
\text { biharmonic spline: } R(r)=r
$$

$$
\text { thin plate spline: } R(r)=r^{2} \log (r)
$$




$$
\begin{gathered}
\text { Gaussian: } R(r)=\mathrm{e}^{-c r^{2}} \\
\text { triharmonic spline: } R(r)=r^{3} \\
\text { multiquadratic (for topographical data): } R(r)=\sqrt{r^{2}+c^{2}} \\
\text { exponential: } R(r)=\mathrm{e}^{r}
\end{gathered}
$$

The parameters $\alpha_{I}$ are obtained by solving the following equations

$$
\left(\begin{array}{cc}
\mathbf{A} & \mathbf{P} \\
\mathbf{P}^{\mathrm{T}} & \mathbf{0}
\end{array}\right)\left(\begin{array}{l}
\boldsymbol{\alpha} \\
\boldsymbol{\beta}
\end{array}\right)=\left(\begin{array}{l}
\boldsymbol{\phi} \\
\mathbf{0}
\end{array}\right)
$$

where

$$
\begin{aligned}
& A_{I J}=R\left(\left\|\mathbf{x}^{I}-\mathbf{x}^{J}\right\|\right), I, J=1 \text { to } N, \\
& P_{I j}=x_{j}^{I}, P_{I_{0}}=1, I=1 \text { to } N, j=1 \text { to } n_{\mathrm{SD}}, \\
& \boldsymbol{\alpha}=\left\{\alpha_{I}\right\}, I=1 \text { to } N, \\
& \boldsymbol{\beta}=\left\{\alpha_{I}\right\}, I=-n_{\mathrm{SD}} \text { to } 0 \text { and } \\
& \boldsymbol{\phi}_{I}=\phi\left(\mathbf{x}^{I}\right) .
\end{aligned}
$$

Note that $\phi\left(\mathbf{x}^{I}\right)=0$ except for the off-surface points. The above equations are obtained by setting $\mathbf{x}$ to $\mathbf{x}^{I}$ for each of the surface points in Equation (4). While the above system matrix is both symmetric and positive semi-definite it is almost full; in Carr et al. [8] multipolar techniques make possible the treatment of millions of points. It should be noted that the matrix on the LHS becomes singular when the points are not unique. We have experimented with the biharmonic and exponential radial basis functions.

In generating the volumes for voxels that are cut by a surface, approximate local surface representations are desirable. For this purpose, a local representation by finite element shape functions is used:

$$
\tilde{\phi}(\mathbf{x})=\sum_{I} N_{I}(\mathbf{x}) \phi_{I}
$$

where $\tilde{\phi}(\mathbf{x})$ is the approximation to the surface, $\phi_{I}=\phi\left(\mathbf{x}_{I}\right)$ where $\mathbf{x}_{I}$ are the nodes of the voxel and $N_{I}(\mathbf{x})$ are the shape functions, i.e. the 4-node shape functions for a pixel.

The zero isobar of this approximation is shown along with that of the radial basis functions for an 0.96 radian arc of a circle in Figure 2. It can be seen that the agreement is quite good, though the exponential is better than the biharmonic.

\subsection{Background mesh (voxels)}

The implicit function is enclosed by a bounding box $B$ that includes all points $\mathbf{x}$ such that $\phi(\mathbf{x})<0$. For the time being, we assume that the bounding box is rectangular, but this is not necessary. The bounding box could also consist of unions of rectangular boxes.

An array of voxels sometimes called a background mesh, is then constructed. This method is similar to the background method quadrature described for meshless methods in Belytschko et al. [17]. The selection of the voxel size is a crucial step in the procedure, since it is necessary to determine a voxel size that leads to reasonably economical computations, yet 


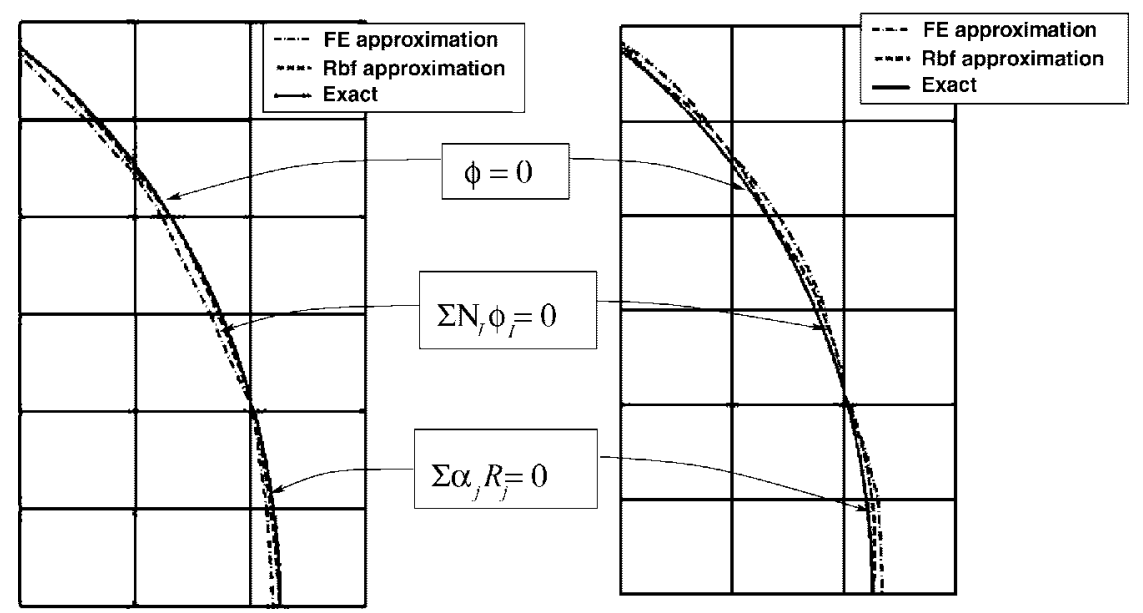

Figure 2. Local approximations of the zero isobar compared with the exact curve for the exponential and biharmonic cases on the left and right, respectively.

corresponds closely enough with the details of the object so that the boundaries of the object in each voxel are simple.

The voxel size must also be such as to achieve to requisite accuracy in the finite element solution without excessive solution time. This involves error estimates, which is a topic outside the scope of this paper, see Babuška and Strouboulis [18]. In most cases, a reasonable voxel size can be set by inspection.

\subsection{Active voxels}

The voxels that are to be active in the finite element model are selected next. This is easily accomplished from the implicit surface function, since at least one node of a voxel must be inside the surface for the voxel to be active. This implies that at least for one node of an active voxel, $\phi\left(\mathbf{x}_{I}\right)<0$. An algorithm for identifying the active voxels is given in Table I.

Table I. Procedure for classifying voxels as active and inactive voxels.

- Compute $\phi\left(\mathbf{x}^{I}\right)$ for all nodes.

- Loop over all voxels, $V=1$ to $N_{V}$.

- loop over nodes of voxels, $I$ to $N$.

- if $\exists$ a node where the value of $\phi_{I}<0 \Rightarrow$ Active voxel.

- if $\phi_{I}<0 \forall$ nodes $\Rightarrow$ Active interior voxel.

- if Active voxel and not interior voxel $\Rightarrow$ Active surface voxel.

- if not an Active voxel $\Rightarrow$ Inactive voxel.

- end loop over voxels. 


\section{FINITE ELEMENT DISCRETIZATION}

The development of the discrete finite element equations of equilibrium is similar to that for the standard finite element method. We will use a structured mesh, but the weak form for a structured mesh is identical to that for an unstructured mesh. To begin, we consider the virtual elements to be voxels and place nodes along the edges and surfaces of all active voxels. This leads to a set of active nodes. An example for 4-node element is shown in Figure 1. As can be seen, active nodes will occur outside the object; these active nodes are associated with active surface voxels. It is of course possible to vary the size of the cells by integer factors in different subdomains or to use different order of elements. In fact, this method is extremely well-suited to $p$-type elements or spectral elements, which tend to perform better when the element surfaces are parallel and orthogonal, e.g. rectangular in two dimensions.

Here, for simplicity we consider small displacement elastostatics. The governing equations are

$$
\nabla \cdot \boldsymbol{\sigma}+\mathbf{b}=0 \quad \text { in } \Omega
$$

where

$$
\begin{aligned}
\boldsymbol{\sigma} & =\mathbf{C}: \boldsymbol{\varepsilon} \\
\boldsymbol{\varepsilon} & =\nabla_{\mathrm{s}} \mathbf{u}
\end{aligned}
$$

where $\boldsymbol{\sigma}$ is the Cauchy stress, $\mathbf{b}$ is the body force per unit volume, $\mathbf{C}$ is Hooke's tensor, $\boldsymbol{\varepsilon}$ is the strain, $\mathbf{u}$ is the displacement and $\nabla_{\mathrm{s}}$ is the symmetric part of the gradient operator.

The essential and natural boundary conditions are, respectively

$$
\begin{aligned}
\mathbf{u}=\overline{\mathbf{u}} & \text { on } \Gamma_{u} . \\
\mathbf{n} \cdot \boldsymbol{\sigma}=\overline{\mathbf{t}} & \text { on } \Gamma_{t}
\end{aligned}
$$

where $\Gamma_{u}$ and $\Gamma_{t}$ are the prescribed displacement and prescribed traction boundaries, respectively.

The space of admissible displacement fields is

$$
u=\left\{\mathbf{u} \in \mathcal{U} \mid \mathbf{u} \text { is smooth and } \mathbf{u}=\overline{\mathbf{u}} \text { on } \Gamma_{u}\right\}
$$

The weak form of the equations of elastostatics is for $\mathbf{u} \in \mathcal{U}$

$$
\int_{\Omega_{a}} \bar{H}(-\phi) \boldsymbol{\varepsilon}(\mathbf{u}) \mathbf{C} \boldsymbol{\varepsilon}(\mathbf{v}) \mathrm{d} \Omega=\int_{\Omega_{a}} \bar{H}(-\phi) \mathbf{b} \cdot \mathbf{v} \mathrm{d} \Omega+\int_{\Gamma_{t}} \overline{\mathbf{t}} \cdot \mathbf{v} \mathrm{d} \Gamma \quad \forall \mathbf{v} \in \mathcal{U}_{0}
$$

where $\Omega_{a}$ is the union of all active voxels, $\bar{H}(\cdot)$ is the standard Heaviside step function and $u_{0}$ is $u$ with $\overline{\mathbf{u}}=0$ on $\Gamma_{u}$. The above can be used directly only when prescribed displacement boundaries coincide with the surface of the voxels. Otherwise we use a Lagrange multiplier method, see for example Belytschko et al. [17].

The displacement field is given by

$$
\mathbf{u}(\mathbf{x})=\sum_{I} N_{I}(\mathbf{x}) \mathbf{u}_{I}
$$


where $\mathbf{u}_{I}$ are the nodal displacements and $N_{I}(\mathbf{x})$ the shape functions (note that we use upper case subscripts for subscripts that pertain to nodes). Substituting the above into the weak form (11) we obtain the standard finite element equations

$$
\sum_{J} \mathbf{K}_{I J} \mathbf{u}_{J}=\mathbf{f}_{I}^{\mathrm{ext}}
$$

where

$$
\begin{aligned}
\mathbf{K}_{I J} & =\int_{\Omega_{a}} \bar{H}(-\phi) \mathbf{B}_{I}^{\mathrm{T}} \mathbf{C B}_{J} \mathrm{~d} \Omega \\
\mathbf{f}_{I}^{\mathrm{ext}} & =\int_{\Omega_{a}} \bar{H}(-\phi) N_{I}(\mathbf{x}) \mathbf{b}(\mathbf{x}) \mathrm{d} \Omega+\int_{\Gamma_{t}} N_{I}(\mathbf{x}) \mathbf{t}(\mathbf{x}) \mathrm{d} \Gamma
\end{aligned}
$$

and $\mathbf{B}$ is the standard strain-displacement matrix that gives $\boldsymbol{\varepsilon}$ by

$$
\boldsymbol{\varepsilon}=\sum_{I} \mathbf{B}_{I} \mathbf{u}_{I}
$$

The numerical quadrature of the weak form (11) is performed voxel by voxel similar to the procedure used in the meshless EFG method [17]. For clarity, we simply consider the quadrature of a scalar $f$; so we have

$$
\int_{\Omega_{a}} \bar{H}(-\phi) f \mathrm{~d} \Omega=\sum_{e=1}^{N_{V}^{\text {act }}} \int_{\Omega_{e}} f \mathrm{~d} \Omega
$$

where $N_{V}^{\text {act }}$ is the number of active voxels.

The quadrature procedure depends on whether a voxel is a surface voxel or an interior voxel. The surface and interior voxels can be segregated during the identification of the active voxels. Quadrature over the interior voxels is straightforward. We use Gaussian quadrature with the number of quadrature points depending on the order of the finite element approximation in the element, e.g. $2 \times 2$ Gauss quadrature for bilinear displacement elements in two dimensions.

For surface voxels, the integration procedure is more involved, since it is necessary to account for the surface that passes through the voxel, i.e. only part of the voxel contributes to $\Omega$; this part is called $\Omega_{e}$. These techniques still require further development, but we describe two methods based on linear approximation to the surface which works well with low order elements. We first describe the methods in two dimensions. In the first method, the integration is performed by cutting each voxel as shown in Figure 3 into two triangles. The finite element

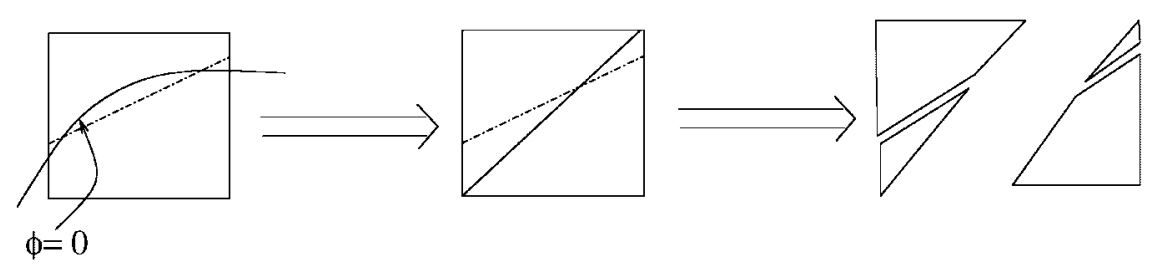

Figure 3. Scheme to subdivide an active voxel for quadrature. 
approximation to the surface function (12), is approximated by a linear function using least squares. Each of these triangles will then be cut by the surface into a quadrilateral and a triangle. Thus, the quadrature over the voxel then consists of quadrature over 2 triangles and 2 quadrilaterals, over which standard quadrature methods are used.

In the second method, the same least square fit is used to subdivide the element into two polygons. These are triangulated by Delaunay triangulation and the quadrature is performed over the elements interior to the surface.

The traction boundary conditions involve quadrature over a curve or surface (in 2D and $3 \mathrm{D}$, respectively). We integrate the traction term over the zero isobar of the finite element approximation of the implicit function (Figure 4) inside each of the surface elements where the traction boundary condition is to be applied.

The integration on the boundary is performed as follows (We present procedure for arbitrary quadrilaterals). We first find $\left(\eta_{1}, \xi_{1}\right)$ and $\left(\eta_{2}, \xi_{2}\right)$, the parental co-ordinates at the intersection of the surface with the finite element; see Figure 4. Consider the integral of $g(\eta, \xi)$;

$$
I=\int_{\Gamma} g(\eta, \xi) \mathrm{d} \Gamma
$$

where $\Gamma$ is the surface according to the finite element approximation (12) of the implicit function given by

$$
\mathrm{d} \Gamma=\left(\mathrm{d} x^{2}+\mathrm{d} y^{2}\right)^{1 / 2}
$$

Note that

$$
\mathrm{d} \phi=\phi_{, x} \mathrm{~d} x+\phi, y \mathrm{~d} y=0
$$

where commas denote partial derivatives. Form (28) it follows that on $\Gamma$

$$
\mathrm{d} y=-\frac{\phi_{, x}}{\phi_{, y}} \mathrm{~d} x \quad \text { or } \quad \mathrm{d} x=-\frac{\phi_{, y}}{\phi_{, x}} \mathrm{~d} y
$$

then

$$
\begin{aligned}
& \mathrm{d} \Gamma=\left[1+\frac{\phi_{, x}^{2}}{\phi_{, y}^{2}}\right] \quad \text { if }\left|\phi_{, y}\right|>\left|\phi_{, x}\right| \\
& \mathrm{d} \Gamma=\left[1+\frac{\phi_{, y}^{2}}{\phi_{, x}^{2}}\right] \quad \text { if }\left|\phi_{, y}\right|<\left|\phi_{, x}\right|
\end{aligned}
$$

The integration over surface voxels for three dimensions is structured so that quadrature formulas are only required for tetrahedra and pentahedra. The hexahedral voxels are first subdivided into tetrahedral subelements as shown in Figure 5. As noted in Usui [19], the hexahedron may be subdivided into tetrahedra in two ways, but the subdivision shown in Figure 5 is a better fit to the volume when the voxel is not a cuboid. Also, when hexahedra in a structured mesh are subdivided into 6 tetrahedra, it is possible to ensure that the triangulation of the contiguous surfaces is identical. For structured meshes, the elements will usually be 


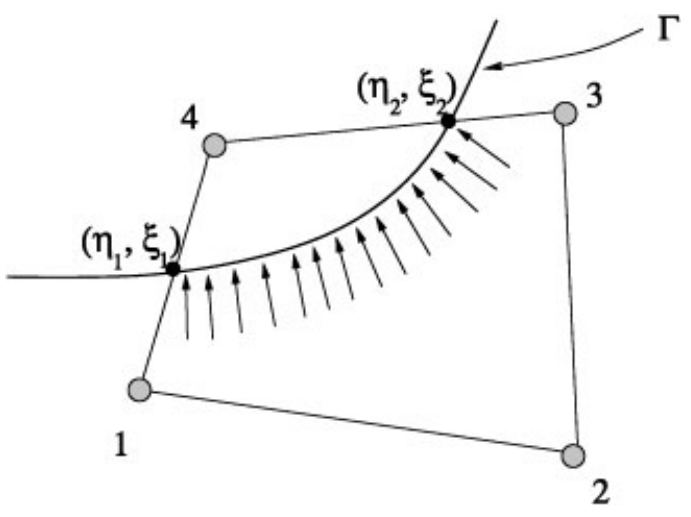

Figure 4. The application of traction over the surface.

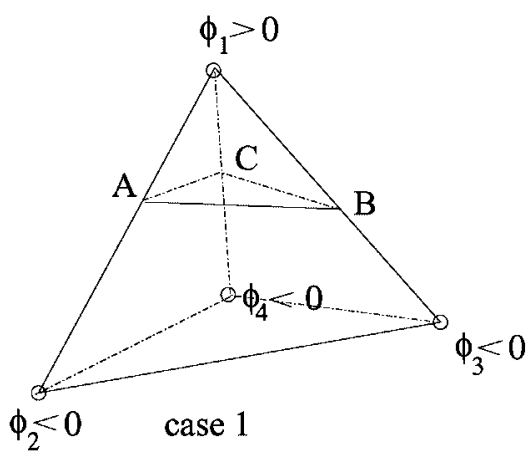

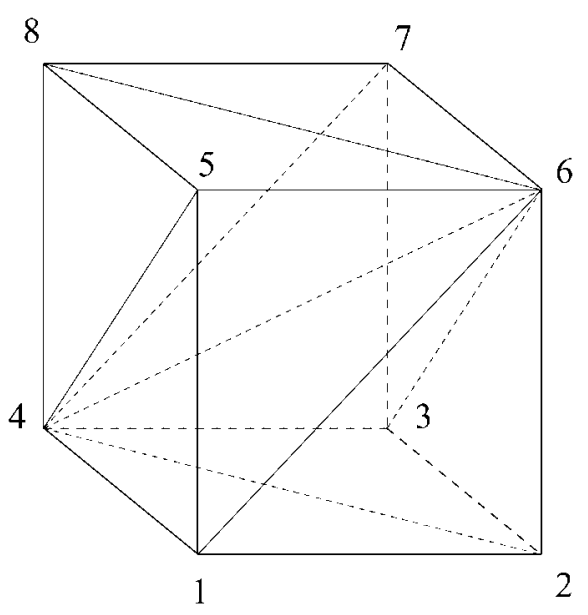

Figure 5. The decomposition of a hexahedron into tetrahedra.

Figure 6. The two possibilities for subdivision of tetrahedra, $\phi_{I} \equiv \phi\left(\mathbf{x}_{I}\right)$.

cuboids, but in some cases structured meshes generated by nonparallel surfaces are desirable. A linear approximation is then obtained for the surface in each tetrahedron.

The tetrahedral subelements are then subdivided into two tetrahedra or a pentahedron and a tetrahedron. The subdivision depends on the values of $\phi_{I}$ at the nodes. We will call a subelement active if it contributes to the Galerkin integrals (22), inactive if it is not.

We first separate out the following:

1. if $\phi_{I} \geqslant 0 \forall I$, then the subelement is inactive.

2. if $\phi_{I} \leqslant 0 \forall I$, then the subelement is active.

Any subelements that do not meet the above fall into one of the following four categories:

Case 1: one $\phi_{I}$ of one sign, three $\phi_{I}$ of another sign; then the volume integral can be obtained from the volumes of tetrahedra (Figure 6(a)). 
Case 2: two $\phi_{I}$ of one sign, two $\phi_{I}$ of another sign; then the tetrahedral subelement consists of two pentahedrons (Figure 6(b)).

Case 3: one $\phi_{I}=0$; then the tetrahedral subelement consists of a pyramid and a tetrahedron.

Case 4: two $\phi_{I}=0$; then the tetrahedral subelement consists of two tetrahedra.

Note that although it would be unusual for $\phi_{I}$ to vanish exactly, successful implementation of the methods described here and for interior features require that $\phi_{I}$ be set to zero when it is smaller than a mesh dependent tolerance, see Moës et al. [5].

The surface integral for inhomogeneous traction boundary conditions is either over a triangle such as $\mathrm{ABC}$ or a quadrilateral such as $\mathrm{ABCD}$; for the surface elements standard quadrature techniques are used to evaluate the boundary integrals of the Galerkin weak form.

\section{INTERNAL DETAILS}

In most engineering and scientific problems, objects that are analysed by finite elements have substantial internal structure. Among these internal details are:

- interfaces between bonded materials

- cracks

- small holes, pores and dislocations

- unbonded interfaces

Some of these examples are illustrated in Figure 7. In the following we describe how some of these features are easily handled in the context of this method. In defining internal surfaces, we again use implicit functions.

Any interface $\Gamma^{\text {int }}$ is described by the zero isobar of an implicit function $\phi^{\text {int }}(\mathbf{x})$; we refer to a single interface for clarity but the technique can be extended to many interfaces. The finite element form of the implicit function is given in terms of nodal values by

$$
\phi^{\text {int }}(\mathbf{x})=\sum_{I} N_{I}(\mathbf{x}) \phi_{I}
$$

or by radial basis functions as before. One useful form of an implicit function is the signed distance function.

$$
\phi^{\text {int }}(\mathbf{x})=\min \left\|\mathbf{x}-\mathbf{x}_{\gamma}\right\| \operatorname{sign}\left(\mathbf{n} \cdot\left(\mathbf{x}_{\gamma}-\mathbf{x}\right)\right)
$$

where $\mathbf{n}$ is a unit normal to the internal surface; its direction is arbitrary.

\subsection{Interfaces between bonded materials}

Examples of interfaces between bonded materials are the interfaces between different constituents of a composite material, the layers of a geotechnical model, materials bonded by glueing and thermal fits. Some of these are described further in the examples.

Across a bonded interface, the displacement field is continuous but the strains are discontinuous. Thus, it is necessary to construct a displacement field that accommodates this discontinuity in the derivative of the displacement. 


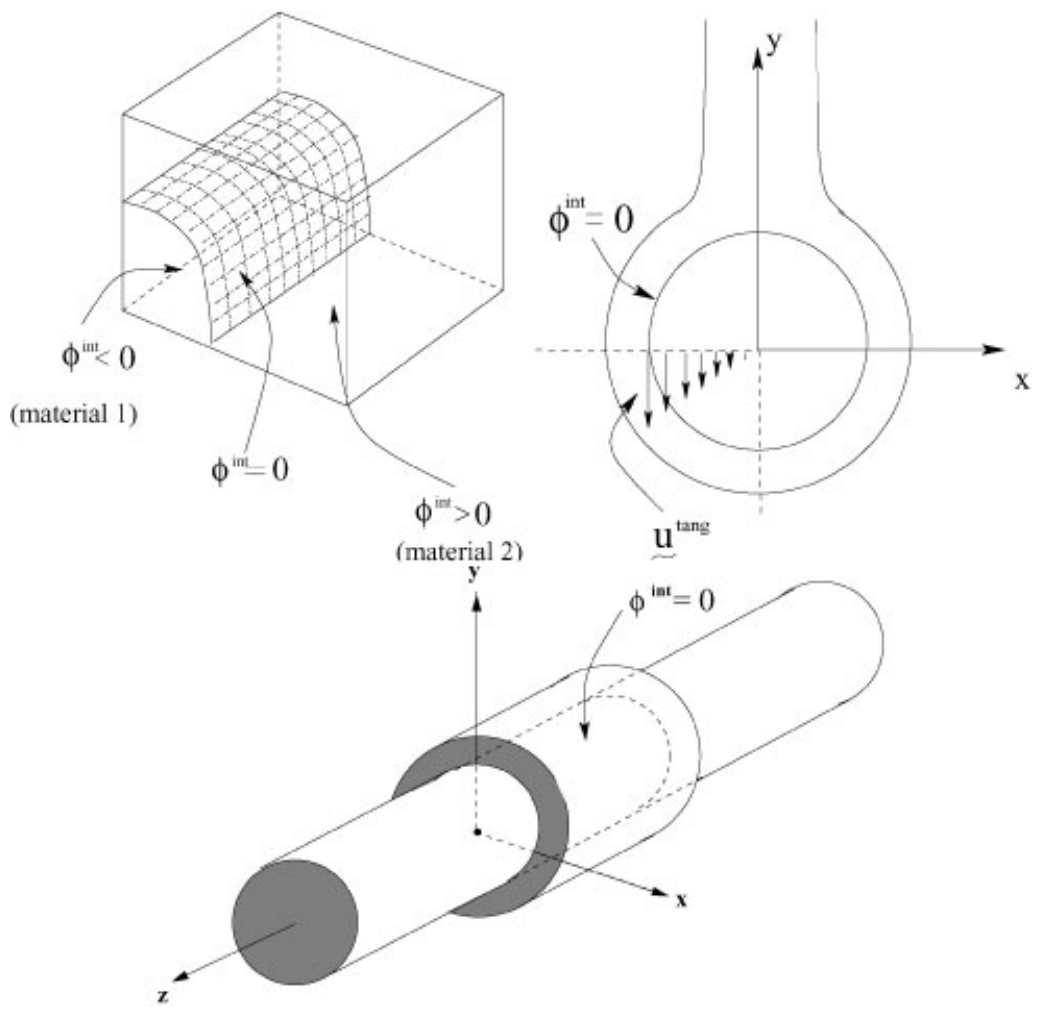

Figure 7. The definition of $\phi^{\text {int }}$ for several examples.

The displacement field in the voxels intersected by the interface are enriched by methods described in Belytschko et al. [1] and Sukumar et al. [10] to model the discontinuous derivative in the displacement across the interface.

For this purpose, we decompose the displacement field

$$
\mathbf{u}=\mathbf{u}^{\text {st }}+\mathbf{u}^{\text {enr }}
$$

where $\mathbf{u}^{\text {st }}$ is the standard finite element field (Equation (20)) and $\mathbf{u}^{\text {enr }}$ is the enrichment field. For a discontinuous derivative the enrichment is

$$
\mathbf{u}^{\mathrm{enr}}=\sum_{I \in C} \mathbf{a}_{I}\left(|\phi|-\left|\phi_{I}\right|\right) N_{I}(\mathbf{x})
$$

where $\mathbf{a}_{I}$ are additional degrees of freedom and $C$ is the set of all nodes whose support is cut by $\Gamma^{\text {int }}$. Note that the term $N_{I}(\mathbf{x})$ localizes the enrichment so that sparsity of the discrete equations is not adversely effected. A special modification described by Sukumar et al. [10] is advantageous.

For voxels cut by $\Gamma^{\text {int }}$, the same procedures used for external boundaries must be used in evaluating the integrals in Equation (23). A major difficulty in the method is presented by intersection of surfaces. When $\Gamma^{\text {int }}$ intersects $\Gamma$ or two interior surfaces intersect within a voxel, the method described previously does not suffice and more complicated schemes are 
needed. At this time, simple mesh generation schemes are recommended, but we have not programmed this.

\subsection{Cracks}

Cracks are among the most common defects that occur in mechanical components. Cracks are associated with singularities in the elastostatic stress fields; the strength of the singularity is the stress intensity factor which determines its rate of growth in cyclic loading. The method described here directly gives the strength of the singularity, i.e. the stress intensity factor, by including the near-tip field as an enrichment.

The crack surface is defined by the implicit function

$$
\phi^{\text {int }}(\mathbf{x})=0
$$

A second implicit function $\psi$ is defined to represent the crack edge. This implicit function is defined so that the intersection of $\psi=0$ with $\phi=0$ gives the crack edge, i.e. $\phi^{\text {int }}(\mathbf{x})=\psi(\mathbf{x})=0$. It is also constructed to be normal to the crack interior levelset function $\phi^{\text {int: }}$

$$
\nabla \psi \cdot \nabla \phi^{\text {int }}=0
$$

Level set techniques (Sethian et al. [20]) can be used to update these functions to model crack growth as described by Stolarska et al. [11] in 2D and Gravouil et al. [21] in 3D.

Across the crack surface, the displacement field is discontinuous and the enrichment models this discontinuity.

To define the displacement field the following nodes need to be identified.

- the set of nodes whose support is completely cut by the crack $(C)$.

- the set of nodes whose support is partially cut by the crack $(P)$.

The enrichment field is given by

$$
\mathbf{u}^{\mathrm{enr}}(\mathbf{x})=\sum_{J \in C} \mathbf{a}_{J} N_{J}(\mathbf{x}) H\left(\phi^{\mathrm{int}}(\mathbf{x})\right)+\sum_{J \in P} \mathbf{c}_{J k} N_{J}(\mathbf{x}) B_{k}(\mathbf{x})
$$

where $\mathbf{a}_{J}$ and $\mathbf{c}_{J k}$ are additional nodal parameters and

$$
H(y)= \begin{cases}+1 & \text { for } y>0 \\ -1 & \text { for } y<0\end{cases}
$$

The asymptotic field near the crack tip is represented by the function $B_{k}$ in Equation (38) where

$$
\left\{B_{k}(r, \theta)\right\}=\left\{\sqrt{r} \sin \left(\frac{\theta}{2}\right), \sqrt{r} \cos \left(\frac{\theta}{2}\right), \sqrt{r} \sin \left(\frac{\theta}{2}\right) \sin (\theta), \sqrt{r} \cos \left(\frac{\theta}{2}\right) \sin (\theta)\right\}
$$

This enrichment scheme is based on Fleming et al. [22].

The angle $\theta$ for the enrichment is defined as

$$
\theta=\tan ^{-1} \frac{\phi^{\text {int }}(\mathbf{x})}{\psi(\mathbf{x})}
$$

More details can be found in Moës et al. [5] and Gravouil et al. [12]. 


\subsection{Sliding interfaces}

Sliding interfaces are an important feature in many engineering problems. For example sliding surfaces are needed between a rod and sleeve, along joints in rock, etc. To model sliding interfaces, the displacement field must be enriched so that discontinuities in the tangential component of the displacement are included in the approximation. This is achieved by enriching the field with a discontinuity in the direction tangential to the sliding surface.

A tangential discontinuity can be constructed by enriching the tangential component of the displacement field with the step function. For example in $2 \mathrm{D}$, we can use

$$
\mathbf{u}^{\mathrm{enr}}(\mathbf{x})=\sum_{I \in C} N_{I}(\mathbf{x}) a_{I} \mathbf{e}_{t}(\mathbf{x}) H\left(\phi^{\mathrm{int}}(\mathbf{x})\right)
$$

where $\mathbf{e}_{t}$ is the tangent to the discontinuity at $\phi^{\text {int }}=0$. In 2D this tangent vector can be defined as $\mathbf{e}_{t}=\mathbf{e}_{z} \times \mathbf{e}_{n}$ where

$$
\mathbf{e}_{n}=\frac{\nabla \phi^{\text {int }}}{\left\|\nabla \phi^{\text {int }}\right\|}
$$

Accuracy can be improved by adding an enrichment based on the nodal values of the tangent (Belytschko et al. [1]). This is done by finding the tangent at the nodes, i.e. in 2D.

$$
\mathbf{u}^{\mathrm{enr}}(\mathbf{x})=\sum_{I \in C} N_{I}(\mathbf{x}) a_{I} \mathbf{e}_{t}\left(\mathbf{x}_{I}\right) H\left(\phi^{\mathrm{int}}(\mathbf{x})\right)
$$

The normal is computed as a nodal average of the normals in the element. The normal is then given by

$$
\mathbf{e}_{n}\left(\mathbf{x}_{I}\right)=\frac{1}{n} \sum_{e=1}^{n} \frac{\nabla \phi^{e}\left(\mathbf{x}_{I}\right)}{\left\|\nabla \phi^{e}\left(\mathbf{x}_{I}\right)\right\|}
$$

where $\nabla \phi^{e}\left(\mathbf{x}_{I}\right)$ is the gradient in element $e$ at node I, where $n$ is the number of nodes in the element.

For three-dimensional problems, two tangent vectors $\mathbf{e}_{t 1}$ and $\mathbf{e}_{t 2}$ are needed. The enrichment is given by

$$
\mathbf{u}^{\mathrm{enr}}(\mathbf{x})=\sum_{I \in C} N_{I}(\mathbf{x}) \sum_{\alpha=1}^{2} \mathbf{a}_{I \alpha} \mathbf{e}_{t \alpha}\left(\mathbf{x}_{I}\right) H\left(\phi^{\mathrm{int}}(\mathbf{x})\right)
$$

\section{RESULTS}

\subsection{Accuracy of surface approximations}

We first give some examples of the accuracy of the surface approximations. The error in this definition is calculated by integrating the value of the function over the exact surface (Table II):

$$
\operatorname{Err}_{\Gamma}=\int_{\Gamma}|\phi(\mathbf{x})| \mathrm{d} \Gamma
$$


Table II. Error in surface definition using different radial basis functions.

\begin{tabular}{lcc}
\hline Number of points & Biharmonic & Exponential \\
\hline Example 1: Error for a circle of radius 3 & \\
4 & 0.0834 & 0.0160 \\
8 & 0.0221 & $4.7369 \times 10^{-4}$ \\
16 & 0.0056 & $1.2609 \times 10^{-4}$ \\
32 & 0.0014 & $3.2046 \times 10^{-5}$ \\
64 & $3.5161 \times 10^{-4}$ & $8.0451 \times 10^{-6}$ \\
128 & $8.7924 \times 10^{-5}$ & $2.0134 \times 10^{-6}$ \\
254 & $2.2333 \times 10^{-5}$ & $5.1151 \times 10^{-7}$ \\
& & \\
Example 2: Error for the geometry shown in Figure 9 \\
30 & 0.0088 & 0.0087 \\
60 & 0.0021 & 0.0021 \\
120 & $5.2433 \times 10^{-4}$ & $5.2572 \times 10^{-4}$ \\
240 & $1.2927 \times 10^{-4}$ & $1.2983 \times 10^{-4}$ \\
480 & $3.2108 \times 10^{-5}$ & $3.2257 \times 10^{-5}$ \\
\hline
\end{tabular}
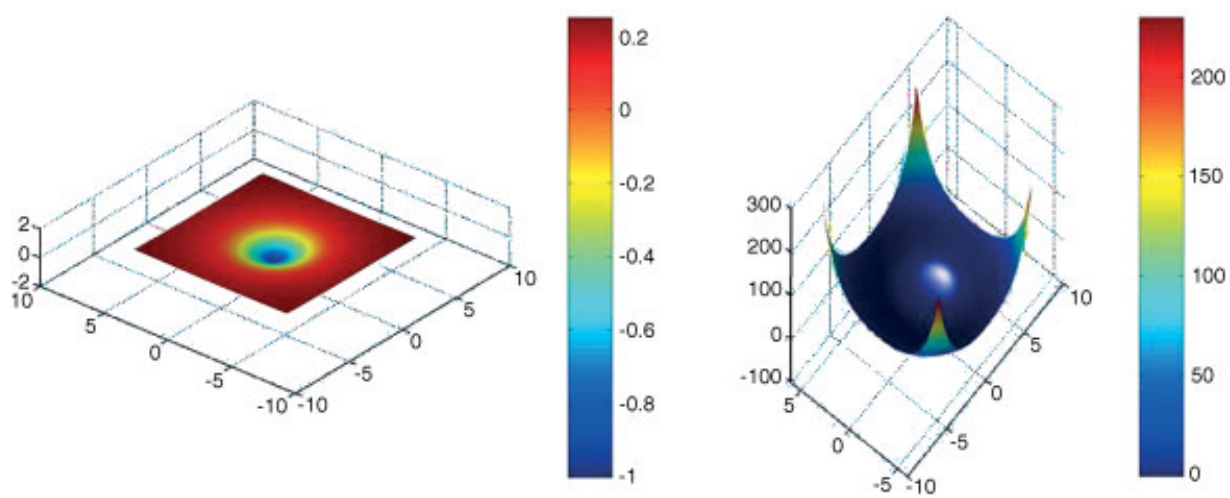

Figure 8 . The implicit function of a circle for the biharmonic and exponential radial basis functions, respectively.

where $\Gamma$ is the exact surface. Different densities of surface points are taken to describe the implicit function, using the radial basis functions defined in Equations (4) and (11). Two shapes were considered;

- a circle

- a quarter piece of a slab with a hole.

The geometry and the contour plot for the second geometry is shown in Figure 9. A quarter space is considered due to axisymmetry.

It can be noted that the errors are comparable for the two types of radial basis functions, although the exponential is more accurate for the circle. Both methods give excellent accuracy with a reasonable number of points. The function is plotted for the two radial basis functions for a circle in Figure 8. It can be seen that even though both the radial basis functions define 

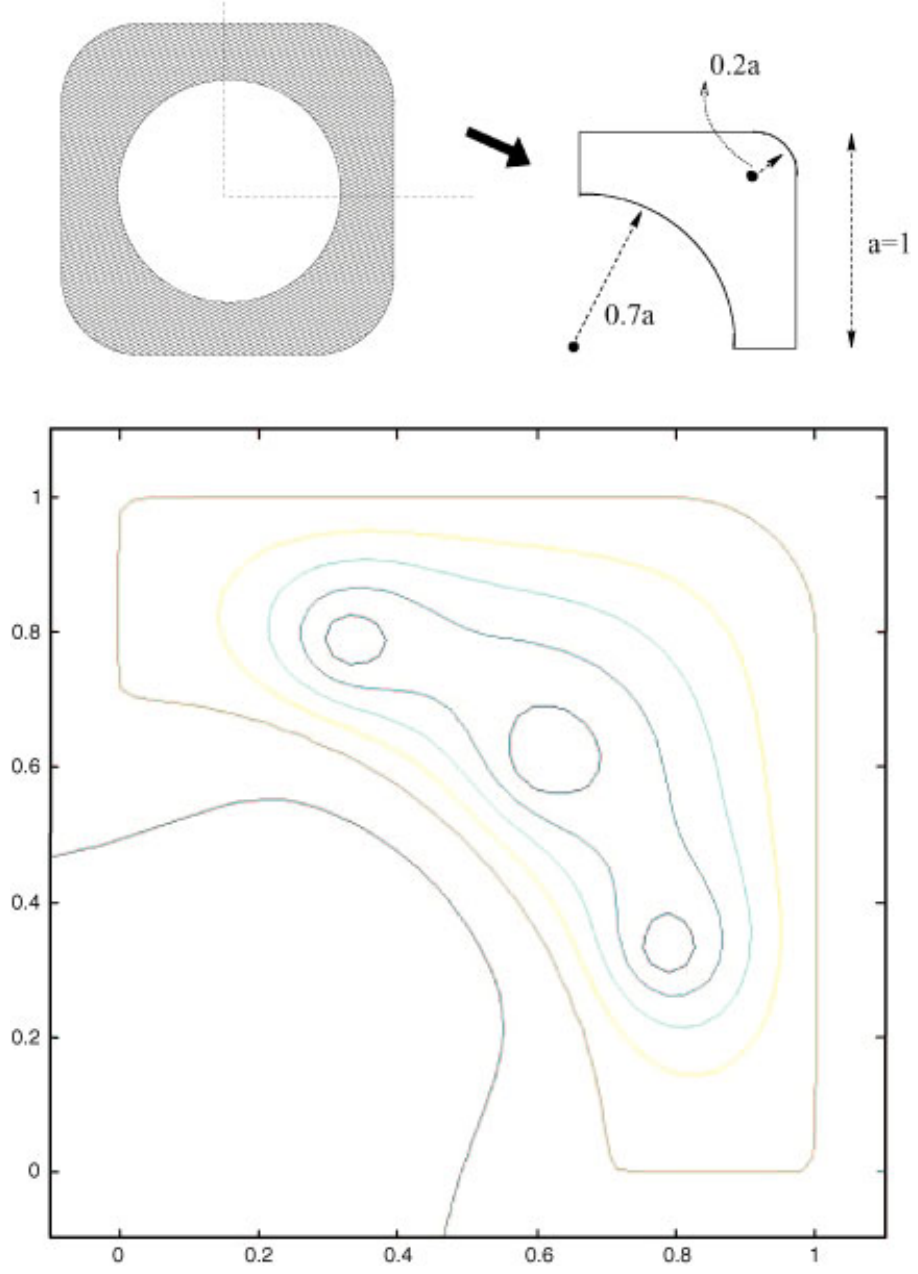

Figure 9. The geometry for example 2 and contour plots of $\phi(\mathbf{x})$.

the shape well and both functions are clearly positive outside the circle, the exponential basis function is steeper and this property can be advantageous. Gaussian radial basis functions, not reported here, can become negative outside the object, which is very undesirable.

\subsection{Examples of complete solutions}

Some standard examples are considered and solved following the schema in Figure 1. The purpose of these examples is to study the effect of the errors in the quadrature of the weak form, particularly the inhomogeneous traction boundary conditions, on the accuracy and rate of convergence.

4.2.1. Hollow cylinder with internal pressure. The first example taken is a hollow cylinder with internal pressure $(p)$. This is modeled as a quarter cylinder due to symmetry with the 

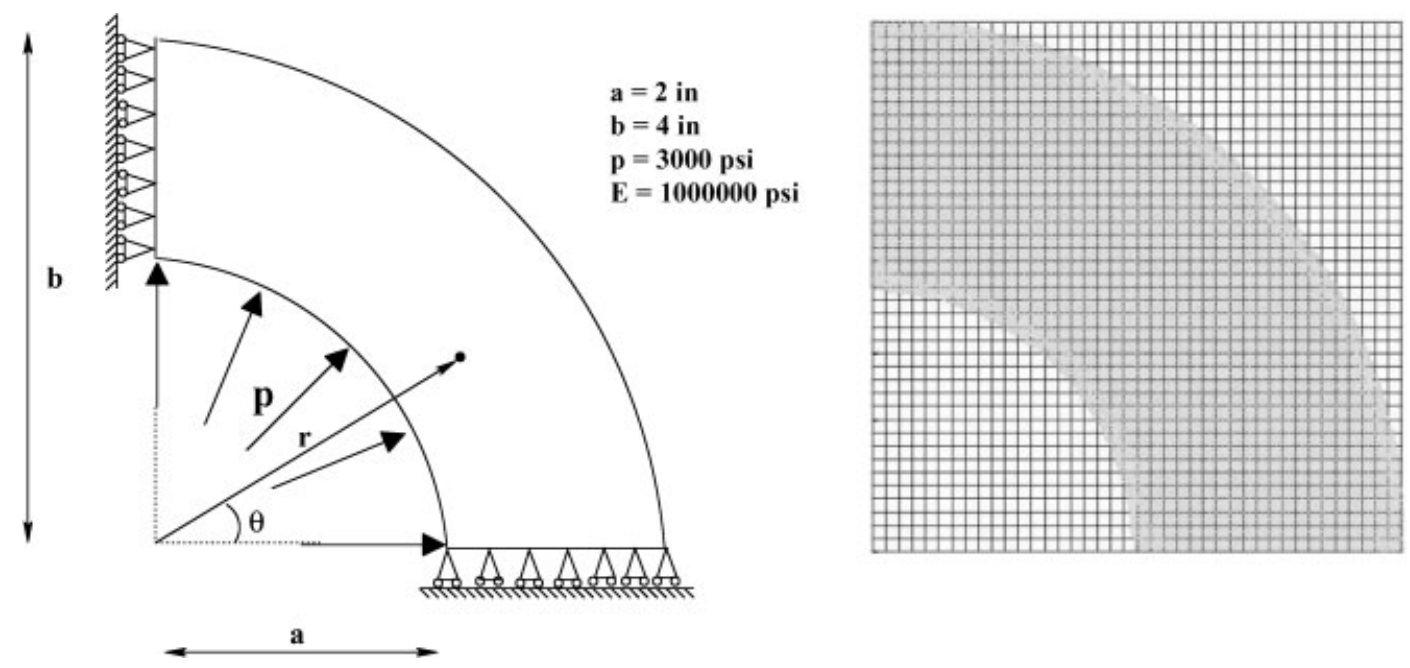

Figure 10. The problem setup for the hollow cylinder problem.

displacement fixed in the normal direction at the two edges as shown in Figure 10. The exact solution for the stress components in polar co-ordinates are as follows [23]

$$
\begin{aligned}
& \sigma_{r}(r, \theta)=\frac{a^{2} p}{b^{2}-a^{2}}\left(1-\frac{b^{2}}{r^{2}}\right) \\
& \sigma_{\theta}(r, \theta)=\frac{a^{2} p}{b^{2}-a^{2}}\left(1+\frac{b^{2}}{r^{2}}\right) \\
& \sigma_{r \theta}(r, \theta)=0
\end{aligned}
$$

where $a$ is the inner radius and $b$ is the outer radius. Note that due to axisymmetry the stresses do not depend on $\theta$. The displacements are as follows:

$$
\begin{aligned}
& u_{r}(r, \theta)=\frac{a^{2} p r}{E\left(b^{2}-a^{2}\right)}\left(1-v+\frac{b^{2}}{r^{2}}(1+v)\right) \\
& u_{r}(r, \theta)=0
\end{aligned}
$$

The parameters chosen in this problem are: $a=2$ in., $b=4$ in., $p=3000$ psi, and plane stress is assumed. The geometry is described by 20 equispaced surface points on each of the two arcs and each of the two straight sides. The surface comprising the geometry shown in Figure 10 is defined using radial basis approximations Equation (4). A biharmonic basis is used in these calculations. The mesh is aligned to the straight edges to facilitate the application of the displacement boundary conditions. It should be noted that the geometry is defined for the quarter cylinder, thereby causing kinks in the surface geometry at the four corners as seen in Figure 10. The radial basis functions seem to capture these kinks rather accurately. 


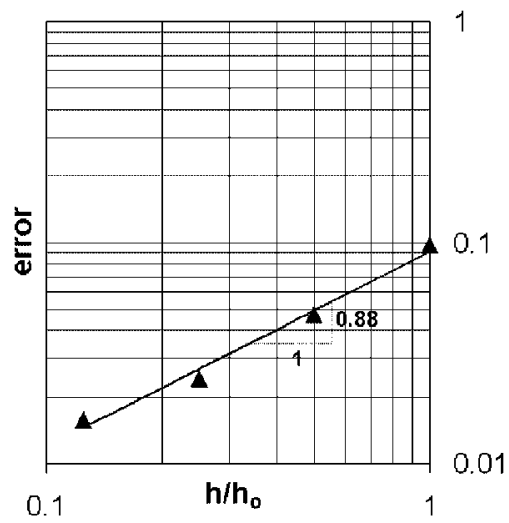

Figure 11. The error in energy for the hollow cylinder problem.

The convergence rate is observed to be 0.88 for the energy norm. The slightly reduced rate of convergence from the optimal rate of 1.0 can probably be attributed to the approximation of the traction boundary condition. As the traction is applied on the zero isobar of the approximation of the surface (Equation (12)), this may effect the accuracy of the solution. Some error may also be attributed to quadrature of Equation (22), but the next example seems to indicate that this error is smaller than error arising from non-zero tractions. The rate of convergence here, incidentally, is not impaired by the number of points that define the geometry. We have run it with 40 points on each segment and obtained almost identical accuracy (Figure 11).

4.2.2. Infinite plate with a hole. The next problem that we consider is that of an infinite plate with a hole of radius $a$ subjected to a uniform tension $\sigma_{0}$ in the $x$-direction (Figure 12). We model this by applying a traction equivalent to the exact stress caused by the uniform traction at infinity on a square plate with a finite dimension. The exact stress field is given by Timoshenko and Goodier [23] and is as follows;

$$
\begin{aligned}
& \sigma_{r}(r, \theta)=\frac{\sigma_{0}}{2}\left(1-\frac{a^{2}}{r^{2}}\right)+\frac{\sigma_{0}}{2}\left(1+\frac{3 a^{4}}{r^{4}}-\frac{4 a^{2}}{r^{2}}\right) \cos 2 \theta \\
& \sigma_{\theta}(r, \theta)=\frac{\sigma_{0}}{2}\left(1+\frac{a^{2}}{r^{2}}\right)-\frac{\sigma_{0}}{2}\left(1+\frac{3 a^{4}}{r^{4}}\right) \cos 2 \theta \\
& \sigma_{r \theta}(r, \theta)=-\frac{\sigma_{0}}{2}\left(1-\frac{3 a^{4}}{r^{4}}+\frac{2 a^{2}}{r^{2}}\right) \sin 2 \theta
\end{aligned}
$$

The radius of the hole, $a=1$ in., Young's modulus $=1000000$ psi, and plane stress was assumed. The hole was described by the radial basis function with 30 surface points. The computations were made with structured meshes ranging from $10 \times 10$ to $80 \times 80$. A convergence study was performed for this problem. The rate of convergence is 0.9524 in the energy norm. The improved rate of convergence compared to the previous problem could be 


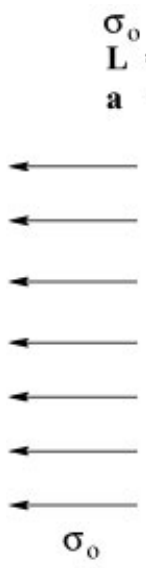

$\sigma_{\mathrm{o}}=1 \mathrm{psi}$

$\mathbf{L}=\mathbf{2}$ in

a $=1$ in
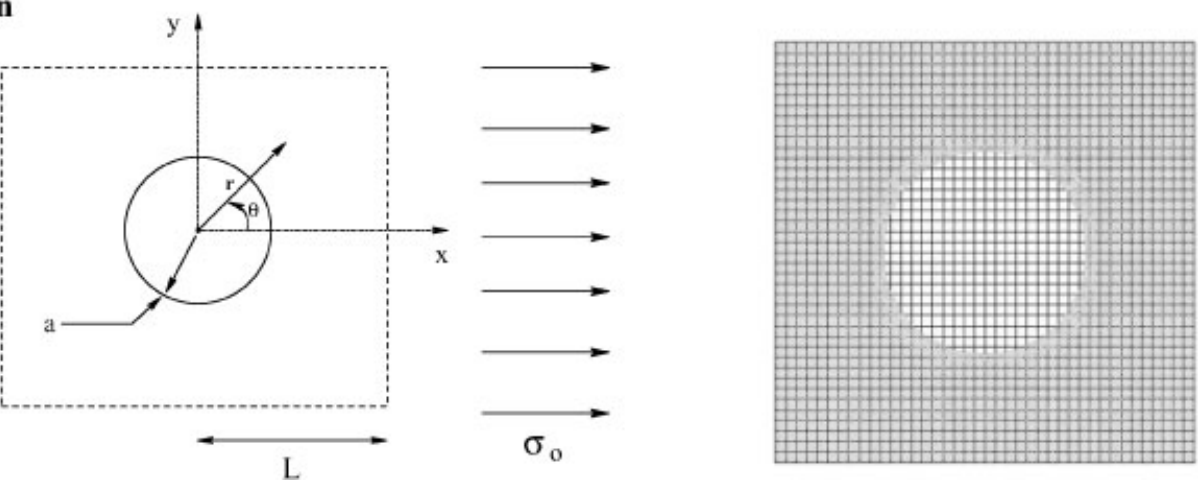

Figure 12. The problem setup for the infinite plate with a hole.

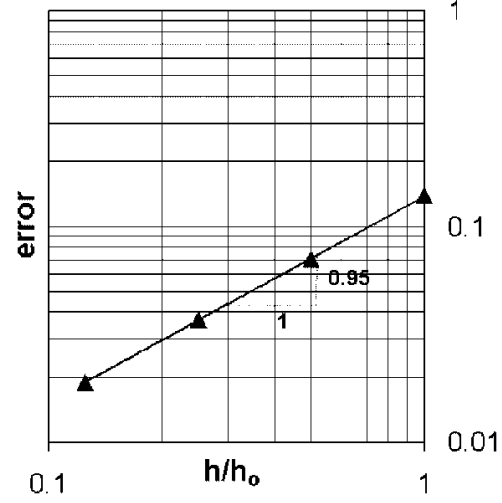

Figure 13. The error in energy for the infinite plate problem.

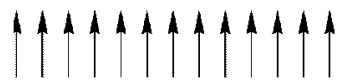

$\mathbf{z}$

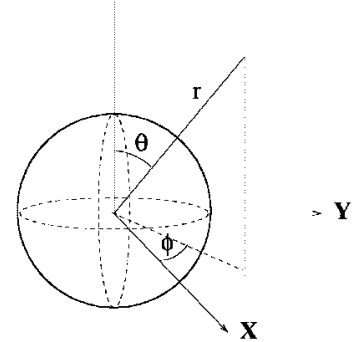

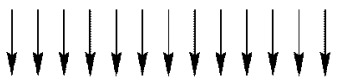

Figure 14. A spherical inclusion in an infinite solid under uniaxial stress.

attributed to the absence of kinks and inhomogeneous traction boundary conditions on the implicit surface (Figure 13).

4.2.3. A spherical inclusion under uniaxial tension. The next few examples illustrate the performance of the method in three dimensional problems for which closed form solutions are available. The primary intent was to study the effect of arbitrary interior interfaces on the accuracy of the method.

The first 3D example is a spherical inclusion under uniaxial tension in an infinite domain is shown in Figure 14. The exact solution for this problem is given by Goodier [24] (see the appendix). 


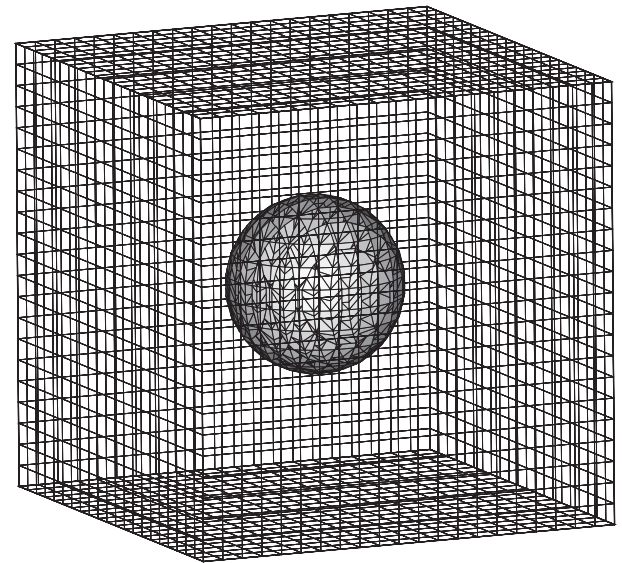

Figure 15. Polygonized material interface with $21 \times 21 \times 21$ mesh.

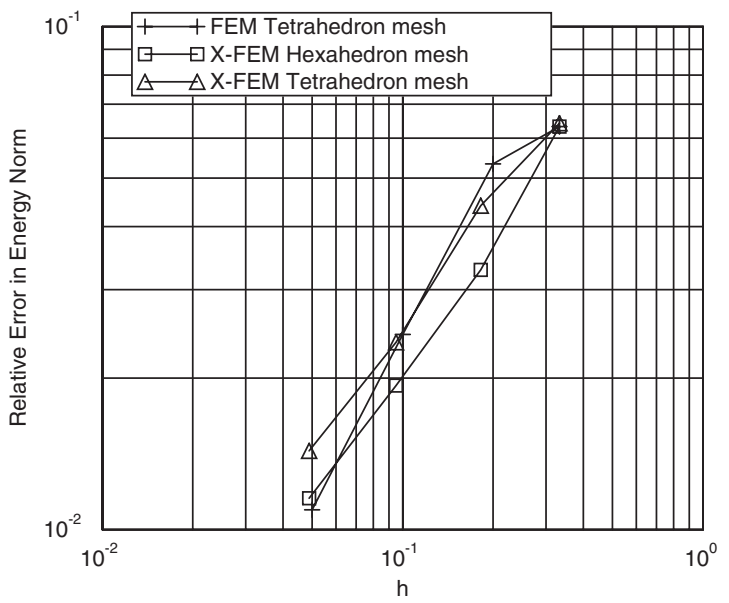

Figure 16. Error in energy for the spherical inclusion problem; structured mesh is labeled $\mathrm{X}$-FEM Hexahedron, conforming mesh is labeled FEM Tetrahedron, X-FEM Tetrahedron is non-conforming unstructured mesh.

In the numerical model, we consider a cubic domain of size $1.0 \times 1.0 \times 1.0$ with an inclusion of radius 0.4 at its center. We impose the exact tractions from the closed form solution to the boundary of the cubic domain, with appropriate constraints added to remove rigid body modes. The material properties are: Young's modulus $E_{1}=2.0$ and $E_{2}=1.0$, Poisson's ratio $v_{1}=v_{2}=0.3$.

The problem was solved with structured meshes consisting of cubic elements. The meshes were $n \times n \times n$, with $n=6,11,21$ and 41. A typical mesh is shown in Figure 15, along with the polygonized material interface. 


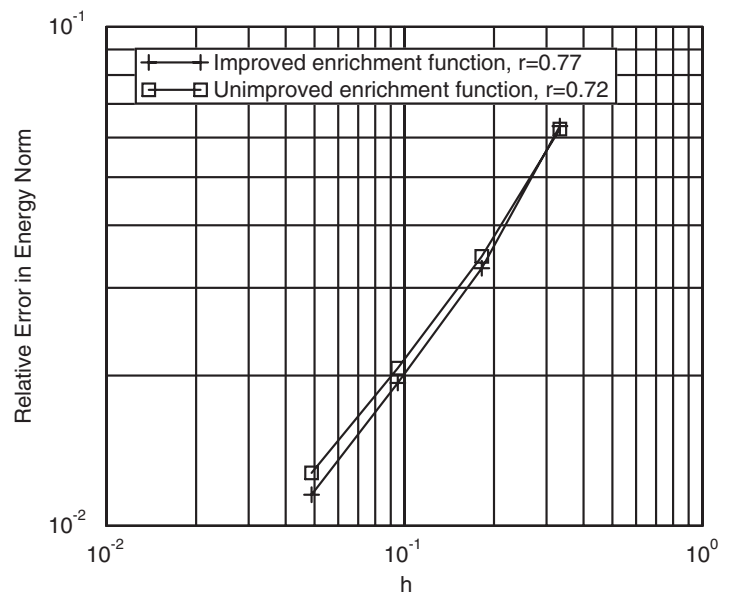

Figure 17. A spherical inclusion in an infinite solid under uniaxial stress with hexahedron mesh.

The error in energy is shown in Figure 16. For comparison, the problem was also solved with a sequence of unstructured tetrahedral meshes. Two types of tetrahedral meshes were used; those conforming to the interface (labeled FEM) and those not conforming to the interface (labeled X-FEM).

As can be seen the accuracy of the structured mesh is better than the unstructured tetrahedral mesh that conforms to the interface, although for the finest mesh the accuracy is the same. The rate of convergence for the structured mesh is about 0.77 , which is slightly below the optimal rate of 1.0. The results for a non-conforming unstructured tetrahedral mesh with enrichment are also shown.

We show the accuracy with the Sukumar et al. [10] correction for discontinuous derivative enrichments, in Figure 17. As can be seen, there is some improvement but not as marked as in two dimensional problems.

4.2.4. 4D carbon-carbon composite. The next example concerns a representative volume element of a composite. In this case the 4D Carbon-Carbon composite of Delenste et al. [25] was considered to show how easy it is to make a mesh for such studies (shown in Figure 18). Representative volume elements are a particularly suitable application of these methods since the domain is cubic and only internal details need to be treated; meshing such problems is very time consuming.

The composite material comprises of four reinforcement directions which are parallel to the diagonals of a cube, i.e. the $(1,1,1),(-1,1,1),(-1,-1,1)$ and $(1,-1,1)$ directions. The implicit functions for the interior surfaces in this case were constructed analytically and directly inserted in the code. For example, for a fibre in the direction given by a unit vector $\mathbf{e}$ with radius $R$, the implicit function deforming a filament that passes through $\mathbf{x}_{0}$ is

$$
\left\|\left(\mathbf{x}-\mathbf{x}_{0}\right) \times \mathbf{e}\right\|^{2}-R^{2}=0
$$




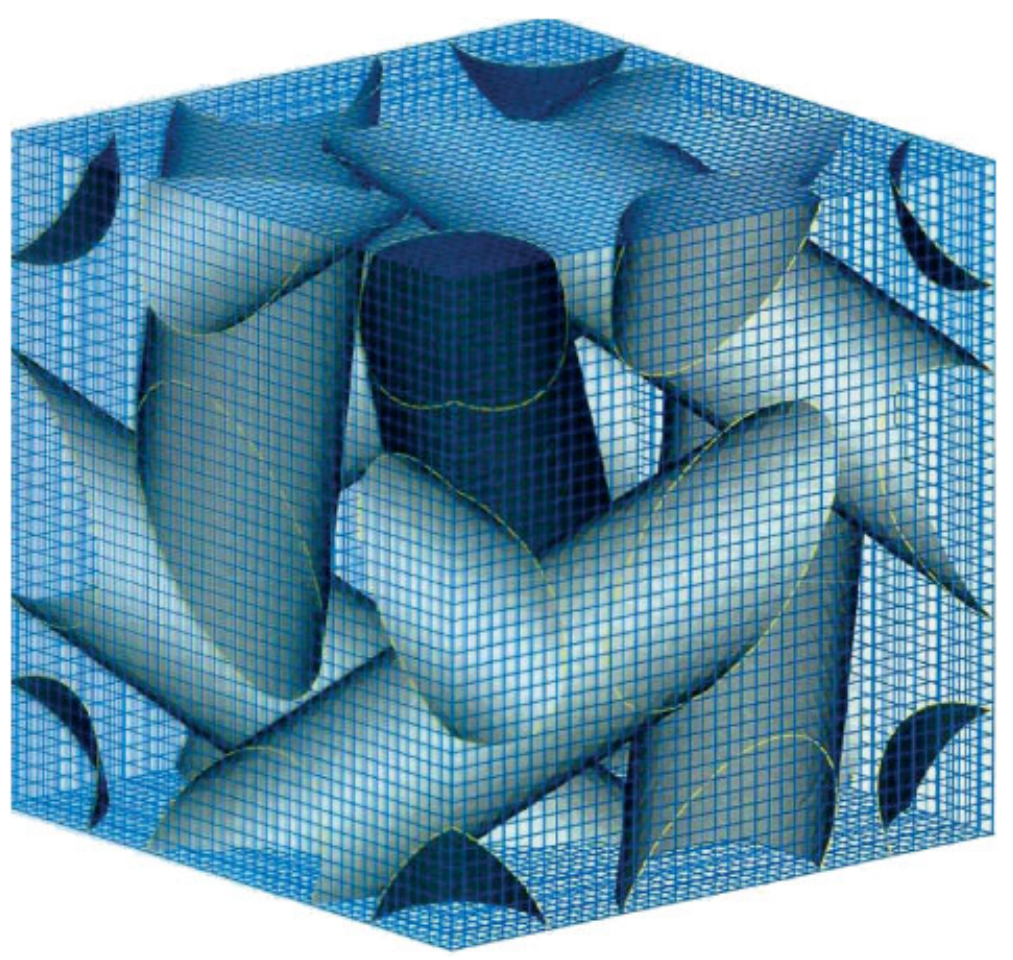

Figure 18. Polygonized material interface of the 4D carbon-carbon composite material in a $21 \times 21 \times 21$ mesh and a yarn volume fraction of 0.49 ; ( $z$ is upward, $x$ is horizontal to right).

The carbon reinforcements are defined as a transverse isotropic material, whose longitudinal Young's modulus is $E_{l}=200.0 \mathrm{GPa}$, longitudinal Poisson's ratio is $v_{l}=0.4$, longitudinal shear modulus is $G_{l}=8.0 \mathrm{GPa}$, transverse Poisson's ratio is $v_{t}=0.3$ and transverse Young's modulus is $10.0 \mathrm{GPa}$. The carbon matrix is defined as an isotropic material with Young's modulus $E=8.0 \mathrm{GPa}$ and Poisson's ratio $v=0.46$. The fibre volume fraction is 0.68 . The values are chosen based on the problem defined in Delenste et al. [25].

The homogenized Young's modulus in the $z$-direction was calculated. The result is $E_{h}=$ 8.5 $\mathrm{GPa}$ which is close to the experimental results $E_{h}=8.0 \mathrm{GPa}$ (see References $[25,29]$ ).

The influences of the ratios of Young's moduli and the volume fraction to the homogenized material constants in $(0,0,1)$ direction were investigated for the material. In this analysis, the yarn and the matrix were defined as isotropic materials. Three cases of yarn volume fractions $(0.67,0.57$ and 0.48$)$ were calculated for different ratio of $E_{\text {matrix }}$ and $E_{\text {yarn }}$, where $E_{\text {matrix }}$ and $E_{\text {yarn }}$ are Young's modulus of matrix and yarn, respectively. Both Poisson's ratio of the matrix and the yarn are 0.4. Yarn volume fraction 0.67 is the maximum volume fraction, since a volume fraction larger than 0.67 causes interpenetration of yarns. The variations of the homogenized Young's modulus and Poisson's ratio are shown in Figures 19 and 20, respectively. 


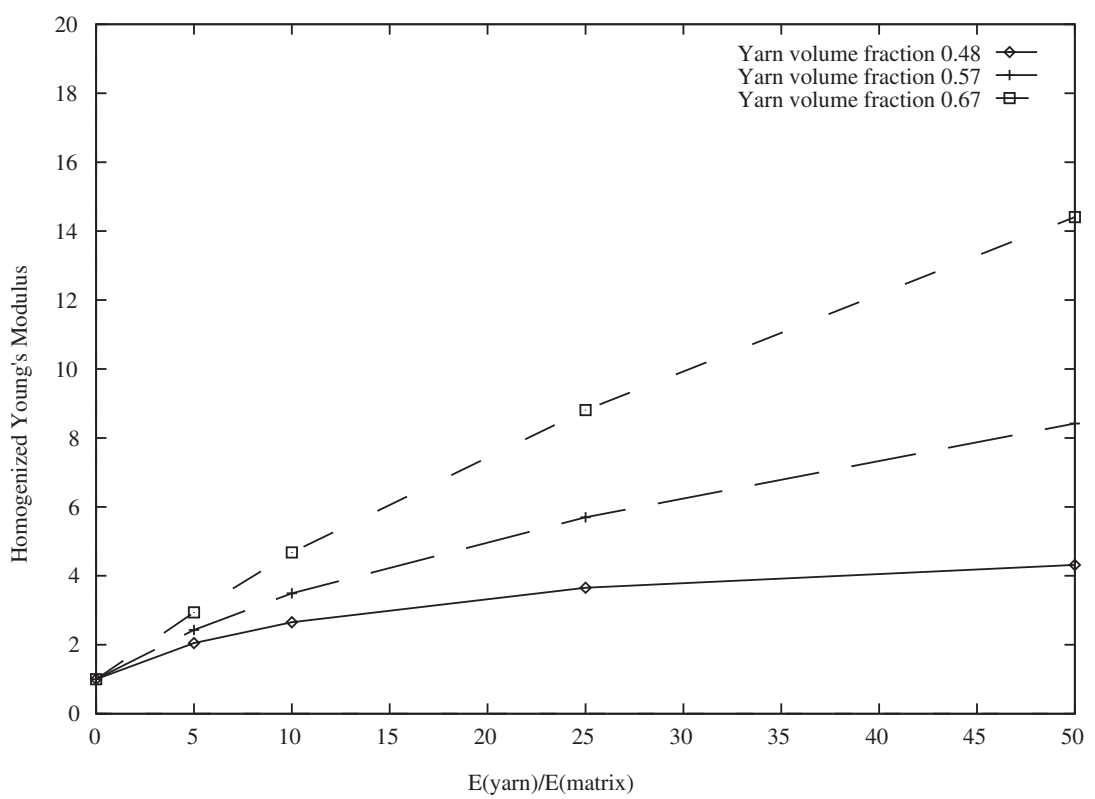

Figure 19. Influence of the $E_{\text {yarn }} / E_{\text {matrix }}$ on homogenized Young's modulus.

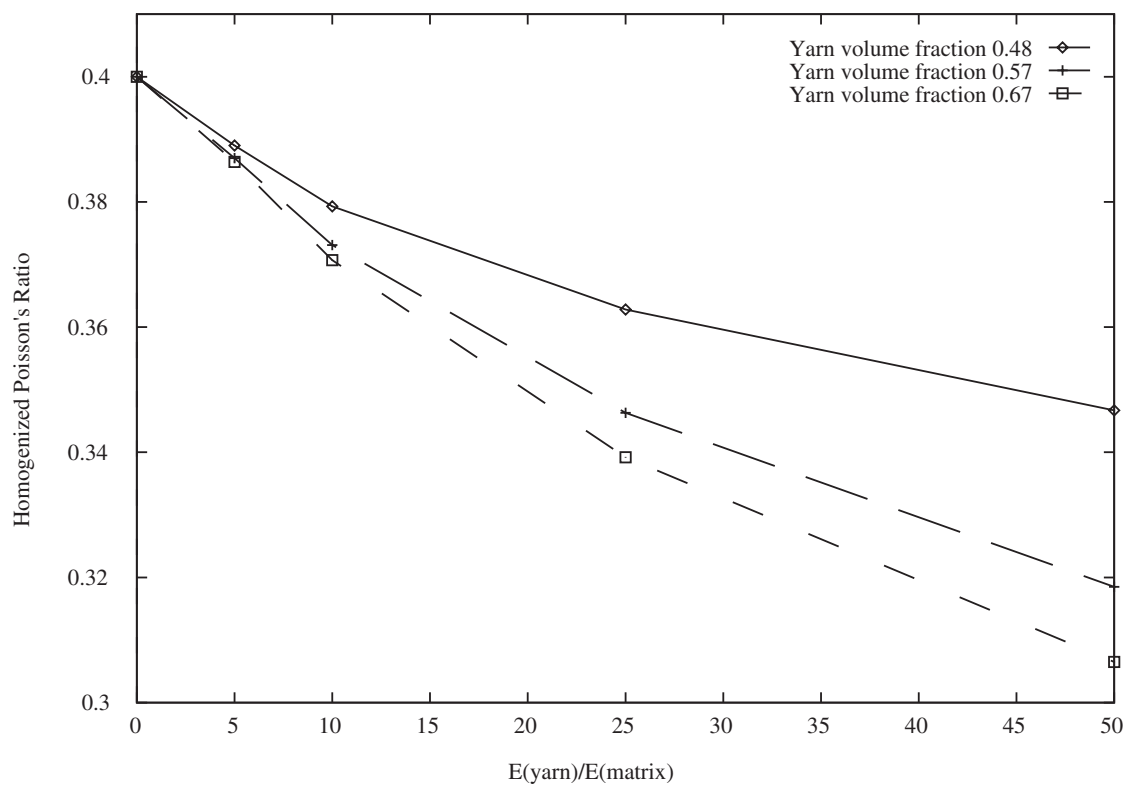

Figure 20. Influence of the $E_{\mathrm{yarn}} / E_{\text {matrix }}$ on homogenized Poisson's ratio. 


\section{CONCLUSIONS}

A simplified method for modelling solid objects by structured finite elements has been presented. The method uses implicit functions to describe the outside surface of the object and any inner surfaces, such as material interfaces, sliding surfaces and cracks. Enrichment functions are then used to modify the structured finite element approximations of the displacement field so that the internal features are modeled. In addition to modelling discontinuities in the displacement field, asymptotic local solutions can be introduced by the enrichment field. Other local fields, such as those for holes, can be introduced as shown by Strouboulis et al. [2]. Overlapping meshes such as described by Charlesworth et al. [26] could also be used. The method is similar to the fictitious element method of Glowinski et al. [27]; the major difference is the introduction of internal details and the description of the geometry by implicit functions.

The method provides good accuracy. Although the rate of convergence is somewhat below the optimal rates, the absolute accuracy is on the same order as for unstructured meshes that observe the discontinuity. In view of the reduced burden in meshing this should be acceptable. Of course, for problems such as crack problems, much greater accuracy can be obtained than with regular finite elements as is apparent from Gravouil et al. [12].

The success of the method hinges on the definition of shapes in implicit radial basis functions. This leads to full equations; but the multipolar methods described by Carr et al. [8] can effectively handle millions of points.

The major drawbacks of the method are the need to perform the integration of the weak form over partitions of an element for active cells cut by boundaries and the fact that the method as developed so far has a uniform mesh over the entire object. The quadrature issue is not difficult when a single surface passes through an element, but becomes awkward when two or more surfaces pass through a single element; such situations are a distinct possibility in multimaterial problems. The quadrature algorithms for such situations could get quite complex, but once coded would not require user-intervention.

The issue of resolution should be tractable by $p$-type methods and structured $h$-type refinement (or coarsening) where the voxel sizes vary in subdomains of the mesh. This would be particularly attractive with adaptive solution strategies, see for example Oden et al. [28].

The main advantage of the method is the simplicity of the model generation. Many models, such as representative volume elements for generating material properties and geotechnical models, are inherently cuboid with considerable internal detail. The generation of unstructured meshes for such problems is quite burdensome; it is time-intensive and it is difficult to ensure quality and correctness. With these methods, the major task becomes the generation of surfaces that define the boundaries of the object and interfaces. Although much remains to be done beyond what has been described here, the potential of the method is quite attractive.

\section{APPENDIX A: THE EXACT SOLUTION FOR SPHERICAL INCLUSION UNDER UNIAXIAL TENSION (GOODIER [24])}

We define a spherical co-ordinate system $(r, \theta, N)$ with its origin at the center of the sphere, whose radius is $a$. A tension $T$ is applied in the $Z$ direction. The problem is symmetric about $\phi$ (Figure 14). The variables are distinguished by superscripts 1 and 2 for the inclusion and 
the matrix, respectively. The exact displacement of the matrix is given by

$$
\begin{aligned}
& u_{r}^{1}=-\frac{A}{r^{2}}-\frac{3 B}{r^{4}}+\left\{\frac{5-4 v_{1}}{1-2 v_{1}} \frac{C}{r^{2}}-\frac{9 B}{r^{4}}\right\} \cos 2 \theta+\frac{T a}{2 E_{1}}\left\{\left(1-v_{1}\right)+\left(1+v_{1}\right) \cos 2 \theta\right\} \\
& u_{\theta}^{1}=-\left(2 \frac{C}{r^{2}}+\frac{6 B}{r^{4}}\right) \sin 2 \theta+-\frac{T a}{2 E_{1}}\left(1+v_{1}\right) \sin 2 \theta
\end{aligned}
$$

The exact displacement of the inclusion is

$$
\begin{aligned}
& u_{r}^{2}=H r+F r+3 F r \cos 2 \theta \\
& u_{\theta}^{2}=-3 F r \sin 2 \theta
\end{aligned}
$$

where $a$ is the sphere radius and the stress field of the matrix is

$$
\begin{aligned}
\sigma_{r r}^{1}= & 2 \mu_{1}\left\{\frac{2 A}{r^{3}}-\frac{2 v_{1}}{\left(1-2 v_{1}\right)} \frac{C}{r^{3}}+\frac{12 B}{r^{5}}+\left(-2 \frac{5-v_{1}}{1-2 v_{1}} \frac{C}{r^{3}}+\frac{36 B}{r^{5}}\right) \cos 2 \theta\right\} \\
& +T \cos \theta \cos \theta \\
\sigma_{\theta \theta}^{1}= & 2 \mu_{1}\left\{-\frac{A}{r^{3}}-\frac{2 v_{1}}{\left(1-2 v_{1}\right)} \frac{C}{r^{3}}-\frac{3 B}{r^{5}}+\left(\frac{C}{r^{3}}-\frac{21 B}{r^{5}}\right) \cos 2 \theta\right\}+T \sin \theta \sin \theta \\
\sigma_{\phi \phi}^{1}= & 2 \mu_{1}\left\{-\frac{A}{r^{3}}-2 \frac{\left(1-v_{1}\right)}{\left(1-2 v_{1}\right)} \frac{C}{r^{3}}-\frac{9 B}{r^{5}}+\left(\frac{3 C}{r^{3}}-\frac{15 B}{r^{5}}\right) \cos 2 \theta\right\}-T \cos \theta \sin \theta \\
\sigma_{r \theta}^{1}= & 2 \mu_{1}\left\{\frac{-2\left(1+v_{1}\right)}{\left(1-2 v_{1}\right)} \frac{C}{r^{3}}+\frac{24 B}{r^{5}}\right\} \sin 2 \theta
\end{aligned}
$$

The stress field in the inclusion is

$$
\begin{aligned}
\sigma_{r r}^{2} & =2 \mu_{2}\left(\frac{1+v_{2}}{1-2 v_{2}} H+F-3 F \cos 2 \theta\right) \\
\sigma_{\theta \theta}^{2} & =2 \mu_{2}\left(\frac{1+v_{2}}{1-2 v_{2}} H+F-3 F \cos 2 \theta\right) \\
\sigma_{\phi \phi}^{2} & =2 \mu_{2}\left(\frac{1+v_{2}}{1-2 v_{2}} H-2 F\right) \\
\sigma_{r \theta}^{2} & =-6 \mu_{2} F \sin 2 \theta
\end{aligned}
$$

where $A, B, C, F, H$ are

$$
A=-\frac{T}{8 \mu_{1}} \frac{\left(\mu_{1}-\mu_{2}\right)\left\{\left(1-2 v_{2}\right)\left(6-5 v_{1}\right) 2 \mu_{1}+\left(3+19 v_{2}-20 v_{1} v_{2}\right) \mu_{2}\right\}}{\left\{\left(7-5 v_{1}\right) \mu_{1}+\left(8-10 v_{1}\right) \mu_{2}\right\}\left\{\left(1-2 v_{2}\right) 2 \mu_{1}+\left(1+v_{2}\right) \mu_{2}\right\}}
$$




$$
\begin{aligned}
& +\frac{T}{4 \mu_{1}} \frac{\left\{\left(1-v_{1}\right)\left(1+v_{2}\right) /\left(1+v_{1}\right)-v_{2}\right\} \mu_{2}-\left(1-2 v_{2}\right) \mu_{1}}{\left(1-2 v_{2}\right) 2 \mu_{1}+\left(1+v_{2}\right) \mu_{2}} a^{3} \\
B= & \frac{T}{8 \mu_{1}} \frac{\mu_{1}-\mu_{2}}{\left(7-5 v_{1}\right) \mu_{1}+\left(8-10 v_{1}\right) \mu_{2}} a^{5} \\
C= & \frac{T}{8 \mu_{1}} \frac{5\left(1-2 v_{1}\right)\left(\mu_{1}-\mu_{2}\right)}{\left(7-5 v_{1}\right) \mu_{1}+\left(8-10 v_{1}\right) \mu_{2}} a^{3} \\
F= & \frac{5}{4}\left(-1+v_{1}\right) \frac{T}{5 \mu_{1} v_{1}-7 \mu_{1}+10 \mu_{2} v_{1}-8 \mu_{2}} \\
H= & -\frac{1}{2}\left(2 v_{1} v_{2}-v_{1}+1-2 v_{2}\right) \\
& \frac{T}{-\mu_{2} v_{1}-\mu_{2} v_{2} v_{1}-2 \mu_{1} v_{1}+4 \mu_{1} v_{2} v_{1} \mu_{2}-\mu_{2} v_{2}-2 \mu_{1}+4 \mu_{1} v_{2}}
\end{aligned}
$$

where $E$ and $v$ are the appropriate Young's modulus and Poisson's ratio, respectively. The shear modulus $\mu$ is given by $\mu=E / 2(1+v)$.

\section{ACKNOWLEDGEMENTS}

The support of the Office of Naval Research and the Army Research Office is gratefully acknowledged.

\section{REFERENCES}

1. Belytschko T, Moës N, Usui S, Parimi C. Arbitrary discontinuities in finite elements. International Journal for Numerical Methods in Engineering 2001; 50(4):993-1013.

2. Strouboulis T, Copps K, Babuška I. The generalized finite element method. Computer Methods in Applied Mechanics and Engineering 2001; 190:4081-4163.

3. Babuška I, Melenk JM. The partition of unity method. International Journal for Numerical Methods in Engineering 1997; 40(4):727-758.

4. Belytschko T, Black T. Elastic crack growth in finite elements with minimal remeshing. International Journal for Numerical Methods in Engineering 1999; 45(5):601-620.

5. Moës N, Dolbow J, Belytschko T. A finite element method for crack growth without remeshing. International Journal for Numerical Methods in Engineering 1999; 46(1):131-150.

6. Dolbow J, Moës N, Belytschko T. Discontinuous enrichment in finite elements with a partition of unity method. Finite Elements in Analysis and Design 2000; 36(3-4):235-260.

7. Strouboulis T, Copps K, Babuška I. The generalized finite element method: an example of its implementation and illustration of its performance. International Journal for Numerical Methods in Engineering 2000; 47(8): 1401-1417.

8. Carr JC, Beatson RK, Cherrie JB, Mitchell TJ, Fright WR, McCallum BC, Evans TR. Reconstruction and representation of 3D objects with radial basis functions. ACM SIGGRAPH 2001, Los Angeles, CA:67-76, 2001.

9. Turk G, Dinh HQ, O’Brien JF, Yngve G. Variational implicit surfaces. Technical Report GIT-GVU-99-15, Georgia Institute of Technology, 1999.

10. Sukumar N, Chopp DL, Moës N, Belytschko T. Modeling holes and inclusions by level sets in the extended finite element method. Computer Methods in Applied Mechanics and Engineering 2001; 190(46-47):6183-6200.

11. Stolarska M, Chopp DL, Moës N, Belytschko T. Modelling crack growth by level sets in the extended finite element method. International Journal for Numerical Methods in Engineering 2001; 51(8):943-960.

12. Moës N, Gravouil A, Belytschko T. Non-planar 3D crack growth by the extended finite element and level sets-Part I: mechanical model. International Journal for Numerical Methods in Engineering 2002; 53(11): 2549-2568. 
13. Chessa J, Smolinski P, Belytschko T. The extended finite element method (XFEM) for solidification problems. International Journal for Numerical Methods in Engineering 2002; 53(8):1959-1977.

14. Chessa J, Belytschko T. The extended finite element method for two-phase fluids. Journal of Applied Mechanics (ASME) 2002, in press.

15. Wagner G, Liu W, Moës N, Belytschko T. The extended finite element method for rigid particles in Stokes flow. International Journal for Numerical Methods in Engineering 2001; 51(3):293-313.

16. Turk G, O’Brien JF. Shape transformation using variational implicit functions. SIGGRAPH'99: 1999; 335-342.

17. Belytschko T, Lu YY, Gu L. Element-free Galerkin method for static and dynamic Fracture. International Journal of Solids and Structures 1995; 32(17-18):2547-2570.

18. Babuška I, Strouboulis T. The Finite Element Method and its Reliability. Clarendon Press: Oxford, 2001.

19. Usui S. Discontinuities in the extended finite element method and beam and shell adaptivity for structural dynamics. Ph.D. Dissertation, Mechanical Engineering, Northwestern University, 2001.

20. Sethian J, Evolution, implementation and application of level set and fast marching methods for advancing fronts. Journal of Computational Physics 2001; 169(2):503-555.

21. Gravouil A, Moës N, Belytschko T. Non-planar 3D crack growth by the extended finite element and level sets-Part II: level set update. International Journal for Numerical Methods in Engineering 2002; 53(11): 2569-2586.

22. Fleming M, Chen YA, Moran B, Belytschko T. Enriched element-free Galerkin methods for crack tip fields. International Journal for Numerical Methods in Engineering 1998; 40:1483-1504.

23. Timoshenko S, Goodier JN. Theory of Elasticity. McGraw-Hill Book Company, Inc: New York, 1934.

24. Goodier JN. Concentration of stress around spherical and cylindrical inclusion and flaws. Journal of Transactions of the ASME 1933; 55:39-44.

25. Delneste L, Perez B. An inelastic finite element model of 4D carbon-carbon composites. AIAA Journal 1983; 21(8):1143-1149.

26. Charlesworth WW, Cox JJ, Anderson DC. The domain decomposition method applied to Poisson's equation in two dimensions. International Journal for Numerical Methods in Engineering 1994; 37:3093-3115.

27. Glowinski R, Pan TW, Periaux J. The fictitious domain method for external incompressible viscous-flow modeled by Navier-Stokes equations. Computer Methods in Applied Mechanics and Engineering 1994; 112:133-148.

28. Oden JT, Demkowicz L, Rachowicz W, Westermann T. Toward a universal $H$-P adaptive finite-element strategy. 2. A posteriori error estimation. Computer Methods in Applied Mechanics and Engineering 1989; 77(1-2): 113-180.

29. Aubard, Cluzel X, Guitard C, Ladevèze P. Damage modeling at two scales for 4D Carbon/Carbon Composites. Computers and Structures 2000; 78:91-93. 